\title{
Existence and stability results on a class of nonlinear Schrödinger equations in bounded domains with Dirichlet boundary conditions
}

\author{
Marco Ghimenti $^{1}$ • Dimitrios Kandilakis ${ }^{2}$. \\ Manolis Magiropoulos ${ }^{3}$
}

Received: 19 January 2015 / Revised: 19 August 2015 / Accepted: 3 September 2015 /

Published online: 15 October 2015

(C) Springer International Publishing AG 2015

\begin{abstract}
Existence of solution, and $L^{2}$ and $H^{1}$ localization results on a class of nonlinear Schrödinger type equations with a bounded nonlinearity are obtained, for a bounded domain and with Dirichlet boundary conditions. The kind of stability under discussion shows that the corresponding solution exhibits features of a solitary wave type.
\end{abstract}

Keywords Nonlinear Schrödinger equation - Stability of solutions · Solitary wave Mathematics Subject Classification $35 \mathrm{Q} 55 \cdot 37 \mathrm{~K} 45$

The first author is partially supported by G.N.A.M.P.A.

\arco Ghimenti

marco.ghimenti@dma.unipi.it

Dimitrios Kandilakis

dimkand@gmail.com

Manolis Magiropoulos

mageir@staff.teicrete.gr

1 Dipartimento di Matematica, Università di Pisa, Largo Buonarroti 1/c, 56127 Pisa, Italy

2 School of Architectural Engineering, Technical University of Crete, University Campus, Akrotiri, 73100 Chania, Greece

3 Department of Electrical Engineering, Technological Educational Institute of Crete, Estavromenos, 71500 Heraklion, Crete, Greece 


\section{Introduction}

We study existence, stability and localization of soliton type solutions for the nonlinear Schrödinger equation (briefly, NLSE) in the semiclassical limit (that is when $h \rightarrow 0^{+}$), for a bounded domain with Dirichlet boundary conditions.

In our framework, the problem takes the form

$$
\begin{array}{rlrl}
i h \frac{\partial \psi}{\partial t} & =-\frac{h^{2}}{2} \Delta \psi+\frac{1}{2 h^{\alpha}} W^{\prime}(|\psi|) \frac{\psi}{|\psi|}+V(x) \psi, \\
\psi(0, x) & =\phi_{h}(x), & x \in \Omega, & \\
\psi(t, x) & =0, & & (t, x) \in \mathbb{R}_{0}^{+} \times \partial \Omega,
\end{array}
$$

where $\psi \in C^{1}\left(\mathbb{R}_{0}^{+}, H_{0}^{1}(\Omega, \mathbb{C})\right), \Omega \subset \mathbb{R}^{N}$ is open and bounded, $N \geqslant 3, \alpha>0$, $\phi_{h}(x) \in H_{0}^{1}(\Omega)$ is a suitable initial datum, and $V$ is an external potential. Conditions for the nonlinear term $W$ and potential $V$ are to be precised and discussed in the subsequent sections.

The NLSE in presence of a potential is largely studied in literature. In particular, it has been extensively investigated the effect of the potential $V$ on the existence and profile of a stationary solution, that is a solution of the form $\psi(t, x)=U(x) e^{-i \omega t / h}$, $\omega=\lambda / 2$, where $U$ solves the equation

$$
-h^{2} \Delta U+\frac{1}{h^{\alpha}} W^{\prime}(U)+2 V(x) U=\lambda U
$$

The first attempt in this direction is the work of Floer and Weinstein [15], for the one dimensional cubic NLSE (with a generalization to higher dimensions and different nonlinearity in [22]) where, by means of a Lyapunov-Schmidt reduction, it is proved that, if $V$ has a nondegenerate minimum, then a stationary solution exists and this solution has a peak located at this minimum. del Pino and Felmer [14] showed that any (possibly degenerate) minimum of $V$ generates a stationary solution. We also mention $[1,19]$, in which similar results are obtained with different techniques. Concerning global methods, in [23] Rabinowitz proved the existence of a stationary solution by means of a Mountain Pass argument. Later, Cingolani and Lazzo [13] proved that the Lusternik-Schnirelmann category of the minimal level of $V$ gives a lower bound for the number of stationary solutions. The topological approach was also adopted in [2], where a more refined topological invariant is used, and in [9], where the presence of a negative potential allows the existence of a solution in the so-called "zero mass" case.

Another interesting feature is the influence of the domain on the stationary NLSE, when $V=0$. In this case, a single-peaked solution can be constructed. In [20], Ni and Wei showed that the least energy solution for the equation

$$
-h^{2} \Delta u+u=u^{p}, \quad u>0,
$$

in $\Omega$ with homogeneous Dirichlet boundary condition has a unique peak, located at a point $P_{h}$ with $d\left(P_{h}, \partial \Omega\right) \rightarrow \max _{\Omega} d(P, \partial \Omega)$ as $h \rightarrow 0^{+}$. Later, Wei [24] proved 
a result that can be viewed as a converse of the aforementioned theorem. Namely, he showed that for any local maximum $P$ of the distance from the boundary $\partial \Omega$, one can construct a single-peaked solution of (2) whose peak tends to $P$ as $h \rightarrow 0^{+}$. The profile of this solution is, up to rescaling, close to the profile of the ground state solution of the limit problem

$$
-\Delta u+u=u^{p}, \quad u>0,
$$

in $\mathbb{R}^{N}$. We also mention [10], in which the existence of a multi-peaked solution of (2) is proved.

In the present work we follow a different approach, incorporating and exploiting ideas found in [4,6,7], where problem (1) had been studied in the whole $\mathbb{R}^{N}$ for both cases: $V=0$ (existence and stability), and $V \neq 0$ (existence, stability and dynamics).

We want to point out two main differences between this paper and [4]. First, dealing with a bounded domain and bounded nonlinearity gives us enough compactness to prove easily an orbital stability result. In particular, our result is also true for positive nonlinearities, which is forbidden in $\mathbb{R}^{N}$ by Pohozaev's theorem.

This difference is less evident in what comes after, since, when dealing with the semiclassical limit, we have to face a limit problem in the whole space $\mathbb{R}^{N}$, which is the same of [4], so we have to reintroduce some hypothesis.

However, our orbital stability result could be used when studying other situations, e.g. fixing $h$ and looking for solitons of prescribed, possibly large, $L^{2}$ norm (the socalled large solitons). If one is interested in these topics, we recommend the nice paper by Noris, Tavares, Verzini [21], which deals with orbital stability for solitons with prescribed $L^{2}$ norms in bounded domains, and references therein. We point out that in [21], the authors work with pure power nonlinearities, so they can have some compactness loss - for $L^{2}$-critical and supercritical powers - even if the domain is bounded. In particular, for critical and supercritical powers they have orbital stability only for small $L^{2}$ norms.

The second point in which the bounded domain marks a difference with the paper [4], is pointed out in Appendix. In fact, when trying to describe dynamics, immediately it appears a repulsive effect of the boundary. The soliton is affected by a force oriented with the inward normal to the boundary. Unfortunately, the value of the force depends on the $D^{1,2}$ norm of the solution on the boundary, so, at the moment, we are not able to give a quantitative estimate of this repulsive force, and further efforts are needed.

According to this line of thought, we have divided the present work into three sections and an appendix. In Sect. 2, existence and orbital stability results are obtained for the case $V=0$, by referring to the related eigenvalue problem

$$
\begin{aligned}
-\Delta U+W^{\prime}(U) & =\lambda U & & \text { in } \quad \Omega, \\
U & \equiv 0 & & \text { on } \partial \Omega,
\end{aligned}
$$

given that a solution $U(x)$ in $H_{0}^{1}(\Omega)$ of (3) results to a solution $\psi=U(x) e^{-i \lambda t / 2}$ of (1) with the initial condition $\psi(0, x)=U(x)$. These results are summarized in Proposition 2.4. One should notice that the relative proofs work without need to impose 
the usual restriction $2<p<2+4 / N$, by relaxing the restriction to $2<p<2^{*}=$ $2 N /(N-2)$ instead. It is the boundedness of the domain that allows us in doing so.

In Sect. 3, where we assume the presence of an external potential, our basic result, obtained by means of a rescaling procedure, is to prove $L^{2}$ localization in the sense that if we start with an initial datum close to a ground state solution $U$ of

$$
\begin{aligned}
-h \Delta U+\frac{1}{h^{\alpha+1}} W^{\prime}(U) & =\frac{\omega}{h^{\alpha+1}} U & & \text { in } \Omega, \\
U & \equiv 0 & & \text { on } \partial \Omega,
\end{aligned}
$$

the corresponding solution of (1) will keep its $L^{2}$ profile along the motion, provided that $h$ is sufficiently small. Here and in what follows, the restriction $2<p<2+4 / N$ is imposed, since we need to face a limit problem in $\mathbb{R}^{N}$.

In Sect. 4, an $H^{1}$ modular localization result is obtained for the case $V \neq 0$, and for both cases: the unbounded and bounded one. When we work on $\mathbb{R}^{N}$, we start with a ground state solution $U_{1}$ of the $\mathbb{R}^{N}$ counterpart of (3), proving that a solution of the $\mathbb{R}^{N}$ counterpart of (1), with initial condition close to $U_{1}$, preserves its basic modular $H^{1}$ profile as time passes, in the sense that given $\varepsilon>0$, for all $t \geqslant 0$, the ratio of the squared $L^{2}$ norm of $\left|\nabla u_{h}(t, x)\right|$ with respect to the complement of a suitable open ball over the squared $L^{2}$ norm of $\left|\nabla u_{h}(t, x)\right|$ with respect to $\mathbb{R}^{N}$ is less than $\varepsilon$ for $h$ sufficiently small, where $u_{h}(t, x)$ is taken by the polar expression of $\psi(t, x)$, namely, $\psi(t, x)=u_{h}(t, x) e^{i s_{h}(t, x)}$. The bounded case is treated by exploiting ideas developed for the $L^{2}$ problem (Sect. 3).

Finally, and as we mentioned before, in Appedix it is described an attempt to study dynamics in the frame of a bounded domain, where we encountered difficulties due to computational complications related to the action of $\nabla V$ on the motion as well as to the repulsive effect of the boundary.

\section{Case $V=0$}

\subsection{Existence}

For simplicity of exposition we assume $h=1$. As it has been already said, the case $V=0$ is related to problem (3), and a solution $u(x)$ in $H_{0}^{1}(\Omega)$ of (3) results to a solution $\psi=u(x) e^{-i \lambda t / 2}$ of (1) with initial condition $\psi(0, x)=u(x)$.

Notice that a minimizer of

$$
J(u)=\int_{\Omega}\left(\frac{1}{2}|\nabla u|^{2}+W(u)\right) d x
$$

on $S_{\sigma}=\left\{u \in H_{0}^{1}(\Omega):\|u\|_{L^{2}(\Omega)}=\sigma\right\}$, for some fixed $\sigma>0$, is a solution of (3) for suitable $\lambda$. Thus we focus on the existence of such a minimizer. We impose on $W$ the following conditions:

(C1) The map $W$ is a $C^{1}$, bounded and even map from $\mathbb{R}$ to $\mathbb{R}$.

(C2) $\left|W^{\prime}(s)\right| \leqslant c|s|^{p-1}, 2<p<2^{*}=2 N /(N-2)$, where $c$ is a suitable positive constant. 
Remark 2.1 Although it is intuitively quite clear the construction of such maps, an easy concrete example is furnished by choosing $W=\sin s^{p}$, for $s \geqslant 0$, and evenly expanding it on $\mathbb{R}$. We also stress the fact that a bounded $W$ is related to the global well-posedness results by Cazenave.

Notice that

$$
-\infty<\mu=\inf _{u \in S_{\sigma}} J(u)
$$

If $\left\{u_{n}\right\}$ is a minimizing sequence in $S_{\sigma}$ for $J(u)$, that is $J\left(u_{n}\right) \rightarrow \mu$, it is evident that $\left\{u_{n}\right\}$ is bounded in $H_{0}^{1}(\Omega)$, thus, up to a subsequence, $u_{n} \rightarrow \bar{u} \in H_{0}^{1}(\Omega)$, and $u_{n} \rightarrow \bar{u}$ in $L^{2}(\Omega)$. The latter implies $\left\|u_{n}\right\|_{L^{2}(\Omega)} \rightarrow\|\bar{u}\|_{L^{2}(\Omega)}$, thus $\bar{u} \in S_{\sigma}$.

Next, we obtain a result similar to [4, Proposition 11].

Proposition 2.2 If $\left\{w_{n}\right\}$ is a minimizing sequence in $S_{\sigma}$ for $J$, that is $J\left(w_{n}\right) \rightarrow \mu$, satisfying the constrained $P-S$ condition, that is, there exists a real sequence $\lambda_{n}$ of Lagrange multipliers such that

$$
-\Delta w_{n}+W^{\prime}\left(w_{n}\right)-\lambda_{n} w_{n}=\sigma_{n} \rightarrow 0
$$

then $\lambda_{n}$ is bounded.

Proof Since, as we saw above, $w_{n}$ is bounded in $H_{0}^{1}(\Omega)$, (4) implies

$$
\left|\int_{\Omega}\left(\left|\nabla w_{n}\right|^{2}+W^{\prime}\left(w_{n}\right) w_{n}-\lambda_{n} w_{n}^{2}\right) d x\right| \leqslant\left\|\sigma_{n}\right\|_{*}\left\|w_{n}\right\|_{H_{0}^{1}(\Omega)} \rightarrow 0
$$

where by $\|\cdot\|_{*}$ is denoted the dual norm for $H_{0}^{1}(\Omega)$. We have

$$
\begin{aligned}
\int_{\Omega}\left(\left|\nabla w_{n}\right|^{2}\right. & \left.+W^{\prime}\left(w_{n}\right) w_{n}-\lambda_{n} w_{n}^{2}\right) d x \\
& =\int_{\Omega}\left(\left|\nabla w_{n}\right|^{2}+2 W\left(w_{n}\right)-2 W\left(w_{n}\right)+W^{\prime}\left(w_{n}\right) w_{n}-\lambda_{n} w_{n}^{2}\right) d x \\
& =2 J\left(w_{n}\right)-\lambda_{n} \sigma^{2}+\int_{\Omega}\left(W^{\prime}\left(w_{n}\right) w_{n}-2 W\left(w_{n}\right)\right) d x \rightarrow 0
\end{aligned}
$$

Notice that $J\left(w_{n}\right)$ is bounded, and because of condition (C2),

$$
\begin{aligned}
\left|\int_{\Omega}\left(W^{\prime}\left(w_{n}\right) w_{n}-2 W\left(w_{n}\right)\right) d x\right| & \leqslant \int_{\Omega}\left|W^{\prime}\left(w_{n}\right) w_{n}\right| d x+2 \int_{\Omega}\left|W\left(w_{n}\right)\right| d x \\
& \leqslant c_{1}\left\|w_{n}\right\|_{H_{0}^{1}(\Omega)}^{p}+2 k \operatorname{meas}(\Omega)<+\infty,
\end{aligned}
$$

where $k$ is an upper bound of $|W|$. Thus $\lambda_{n}$ is bounded. 
By Ekeland's principle, if $\left\{u_{n}\right\}$ is a minimizing sequence in $S_{\sigma}$ for $J(u)$, we may assume that it satisfies the constrained P-S condition, that is, there exists a real sequence $\lambda_{n}$ such that (4) holds. Because of Proposition 2.2, $\lambda_{n}$ is bounded, and the following hold:

$$
\begin{array}{ll}
\lambda_{n} \rightarrow \lambda, & \\
u_{n} \rightarrow u & \text { in } H_{0}^{1}(\Omega), \\
u_{n} \rightarrow u & \text { in } L^{p}(\Omega) \text { for } 1 \leqslant p<2^{*} .
\end{array}
$$

We have already shown that $u \in S_{\sigma}$. Thus, $u \neq 0$. Next, we show that

$$
-\Delta u+W^{\prime}(u)=\lambda u \text {. }
$$

To this end, if $\varphi$ is a test function, combining the three considerations above with condition (C2), we have

$$
\begin{aligned}
\int_{\Omega} \nabla u_{n} \nabla \varphi d x \rightarrow & \int_{\Omega} \nabla u \nabla \varphi d x, \quad \int_{\Omega} W^{\prime}\left(u_{n}\right) \varphi d x \rightarrow \int_{\Omega} W^{\prime}(u) \varphi d x \\
& \lambda_{n} \int_{\Omega} u_{n} \varphi d x \rightarrow \lambda \int_{\Omega} u \varphi d x,
\end{aligned}
$$

implying (5).

Notice next that due to condition (C2), the Nemytskii operator

$$
W: L^{t}(\Omega) \rightarrow L^{1}(\Omega), \quad 2<t<2^{*},
$$

is continuous, whereas $u_{n} \rightarrow u$ in $L^{t}(\Omega)$, for $2<t<2^{*}$. Thus,

$$
\mu \leqslant J(u)=\frac{1}{2} \int_{\Omega}|\nabla u|^{2} d x+\int_{\Omega} W(u) d x \leqslant \lim _{n \rightarrow \infty} J\left(u_{n}\right)=\mu,
$$

proving that $J(u)=\mu$.

This completes the proof for the existence of a nontrivial solution of (3) for suitable $\lambda$. In fact, the weak convergence $u_{n} \rightarrow u$ turns out to be a strong one: Since $J\left(u_{n}\right)-$ $J(u), \int_{\Omega}\left(W\left(u_{n}\right)-W(u)\right) d x \rightarrow 0$, we obtain $\left\|u_{n}\right\|_{H_{0}^{1}(\Omega)} \rightarrow\|u\|_{H_{0}^{1}(\Omega)}$, thus proving that $u_{n} \rightarrow u$ in $H_{0}^{1}(\Omega)$. Since $W$ has been assumed even, we may take a nontrivial nonnegative solution of (3). By Harnack's inequality, this solution is strictly positive on $\Omega$. We thus obtain a positive solution $\bar{u} \in S_{\sigma}$ for problem (3) for suitable $\lambda$. The wave function

$$
\psi(t, x)=\bar{u}(x) e^{-i \omega t}, \quad \omega=\frac{\lambda}{2}
$$

is a stationary solution of (1), for $h=1, V \equiv 0$, with initial condition $\phi(x)$ $=\psi(0, x)=\bar{u}(x)$. Evidently, $-\bar{u}(x) e^{-i \omega t}, \omega=\lambda / 2$, is a stationary solution of (1), too. 


\subsection{Stability}

We turn next our attention to the stability of the stationary solution. To this end, we focus on the reduced form of (1),

$$
\begin{array}{rlrl}
2 i \frac{\partial \psi}{\partial t} & =-\Delta \psi+W^{\prime}(|\psi|) \frac{\psi}{|\psi|}, & (t, x) \in \mathbb{R}_{0}^{+} \times \Omega, \\
\psi(0, x) & =\phi(x), & x \in \Omega, & \\
\psi(t, x) & =0, & (t, x) \in \mathbb{R}_{0}^{+} \times \partial \Omega,
\end{array}
$$

by taking, as it was mentioned above, $h=1$. The different time slices $\psi_{t}(x)$ of each solution of (6), where such a solution may be understood as the time evolution of some initial condition $\psi_{0}(x)$, could be thought of as elements of a proper phase space $X \subset L^{2}(\Omega, \mathbb{C})$, with the set

$$
\Gamma=\left\{u(x) e^{i \theta}: \theta \in \frac{\mathbb{R}}{2 \pi \mathbb{Z}}, u \in S_{\sigma}, J(u)=\mu=\inf _{w \in S_{\sigma}} J(w)\right\}
$$

being an invariant (under evolution) manifold of $X$. Evidently, $\pm \bar{u}(x) \in \Gamma$.

To make the description of all this more clear, one should notice that if $\psi_{t_{0}}(x)$ is a time slice of a solution $\psi(t, x)$ of $(6)$, the evolution map is defined by

$$
U_{t} \psi_{t_{0}}(x)=\psi_{t_{0}+t}(x)
$$

meaning that this time slice might be considered as the initial condition of the solution $\psi_{1}(t, x)=\psi\left(t+t_{0}, x\right)$. Now, if $u(x) e^{i \theta} \in \Gamma$, then $u$ is a solution of (3) with suitable $\lambda$, and, at the same time, $u(x) e^{i \theta}$ is the initial condition of the solution $\psi(t, x)=$ $u(x) e^{i(\theta-\lambda t / 2)}$ of (6). Since $u(x) e^{i(\theta-\lambda t / 2)} \in \Gamma$ for each $t \geqslant 0$, the invariance of $\Gamma$ follows. We are going to prove orbital stability of $\bar{u}(x) e^{-i \omega t}, \omega=\lambda / 2$, following the definition of orbital stability found in [12], meaning that $\Gamma$ is stable in the following sense: for all $\varepsilon>0$ there exists $\Delta>0$ such that if $\psi(t, x)$ is a solution of (6) satisfying $\inf _{\widetilde{w} \in \Gamma}\|\psi(0, x)-\widetilde{w}\|_{H_{0}^{1}(\Omega)}<\Delta$, then for all $t \geqslant 0$

$$
\inf _{\widetilde{w} \in \Gamma}\|\psi(t, x)-\widetilde{w}\|_{H_{0}^{1}(\Omega)}<\varepsilon .
$$

In Lemmas 3.8 and 3.9, we will see how the orbital stability result leads to a precise description of the modulus of a solution $\psi(t, x)$ starting from a suitable initial datum. It is the need to stretch the localization results described there that made us adopt the proof through polar decomposition that follows rather than the more straightforward approach in [12] (see also Remark 2.3).

Notice that $\Gamma$ is bounded in $H_{0}^{1}(\Omega)$, since for each of its elements $\widetilde{w}=w(x) e^{i \theta}$, $w(x)$ is a constrained minimizer of $J$, whereas $W$ is bounded. Notice that we may take $w(x)>0$.

Suppose $\Gamma$ is not stable. Then there exist $\varepsilon>0$, sequences $\Delta_{n} \rightarrow 0^{+}, \psi_{n}(t, x)$ solutions of (6) and $t_{n} \geqslant 0$ such that 


$$
\inf _{\widetilde{w} \in \Gamma}\left\|\psi_{n}(0, x)-\widetilde{w}\right\|_{H_{0}^{1}(\Omega)}<\Delta_{n}, \quad \inf _{\widetilde{w} \in \Gamma}\left\|\psi_{n}\left(t_{n}, x\right)-\widetilde{w}\right\|_{H_{0}^{1}(\Omega)} \geqslant \varepsilon .
$$

Notice that the first inequality in (8) implies

$$
\inf _{\widetilde{w} \in \Gamma}\left\|\psi_{n}(0, x)-\widetilde{w}\right\|_{L^{2}(\Omega)}<C \Delta_{n} \rightarrow 0,
$$

where $C$ is the Sobolev constant satisfying $\|\cdot\|_{L^{2}(\Omega)} \leqslant C\|\cdot\|_{H_{0}^{1}(\Omega)}$. Thus, we may obtain a sequence $\widetilde{w}_{n}$ in $\Gamma$ such that

$$
\left\|\psi_{n}(0, x)-\widetilde{w}_{n}\right\|_{L^{2}(\Omega)} \rightarrow 0 .
$$

We express now $\psi_{n}(t, x)$ in polar form, namely, $\psi_{n}(t, x)=u_{n}(t, x) e^{i s_{n}(t, x)}$, with $u_{n}(t, x)=\left|\psi_{n}(t, x)\right|, t \geqslant 0$. Since $\left\|\widetilde{w}_{n}\right\|_{L^{2}(\Omega)}=\sigma$, (9) implies that $u_{n}(0, x)$ is bounded in $L^{2}(\Omega)$, and at least up to a subsequence, still denoted by $u_{n}(0, x)$, $\left\|u_{n}(0, x)\right\|_{L^{2}(\Omega)} \rightarrow M \geqslant 0$. Rewriting (9) in its squared form, and taking into consideration that

$$
\int_{\Omega} u_{n}(0, x)\left|w_{n}(x)\right| d x \leqslant\left\|u_{n}(0, x)\right\|_{L^{2}(\Omega)} \sigma
$$

we take

$$
0 \geqslant M^{2}-2 M \sigma+\sigma^{2}=(M-\sigma)^{2},
$$

thus obtaining $M=\sigma$.

The polar form $\psi(t, x)=u(t, x) e^{i s(t, x)}$ turns (6) into the system

$$
\begin{aligned}
-\frac{\Delta u}{2}+\frac{W^{\prime}(u)}{2}+\left(\frac{\partial s}{\partial t}+\frac{1}{2}|\nabla s|^{2}\right) u & =0, & & (t, x) \in \mathbb{R}_{0}^{+} \times \Omega, \\
\partial_{t} u^{2}+\nabla \cdot\left(u^{2} \nabla s\right) & =0, & & (t, x) \in \mathbb{R}_{0}^{+} \times \Omega, \\
u(0, x) e^{i s(0, x)} & =\phi(x), & & x \in \Omega, \\
u(t, x) & =0, & & (t, x) \in \mathbb{R}_{0}^{+} \times \partial \Omega,
\end{aligned}
$$

with the two equations of (10) being the Euler-Lagrange equations of the action functional

$$
\begin{aligned}
A(u, s)=\frac{1}{4} \iint|\nabla u|^{2} d x d t & +\frac{1}{2} \iint W(u) d x d t \\
& +\frac{1}{2} \iint\left(\frac{\partial s}{\partial t}+\frac{1}{2}|\nabla s|^{2}\right) u^{2} d x d t .
\end{aligned}
$$

The total energy is given by

$$
E(\psi)=E(u, s)=\int_{\Omega}\left(\frac{1}{2}|\nabla u|^{2}+\frac{1}{2} u^{2}|\nabla s|^{2}+W(u)\right) d x,
$$


that is,

$$
E(\psi)=E(u, s)=J(u)+\frac{1}{2} \int_{\Omega} u^{2}|\nabla s|^{2} d x .
$$

Independence of time for the energy and for the charge imply that for a solution $\psi(t, x)=u(t, x) e^{i s(t, x)}$ of (6), it holds

$$
\frac{d}{d t} \int_{\Omega} u^{2}(t, x) d x=0, \quad \frac{d}{d t} E(u, s)=0 .
$$

Equivalently, (12) can be expressed as

$$
\begin{aligned}
\|\psi(t, x)\|_{L^{2}(\Omega)} & =\|\phi(x)\|_{L^{2}(\Omega)}, \\
E(\psi(t, x)) & =E(\phi(x))
\end{aligned}
$$

for all $t \geqslant 0$. Noteworthy, for stationary solution, (11) yields $E(\psi)=J(u)$.

Returning to the sequence $\psi_{n}(t, x)$ satisfying (8), we may assume, as we saw, that $\left\|u_{n}(0, x)\right\|_{L^{2}(\Omega)} \rightarrow \sigma$, that is $\left\|u_{n}(t, x)\right\|_{L^{2}(\Omega)} \rightarrow \sigma$, for $t \geqslant 0$, because of (13). We want to show that $\left\{u_{n}\right\}_{n}$ is a minimizing sequence for the functional $J$ on the constraint $\|u\|_{L^{2}(\Omega)}=\sigma$.

One should notice that the first inequality of (8), combined with the boundedness of $\Gamma$ ensure that $\psi_{n}(0, x)$ is bounded in $H_{0}^{1}(\Omega)$. Since $W$ is bounded, (11) ensures that $E\left(\psi_{n}(0, x)\right)$ is bounded, and because of (14), $E\left(\psi_{n}(t, x)\right)$ is bounded, for all $n$ and all $t \geqslant 0$. In particular, $E\left(\psi_{n}\left(t_{n}, x\right)\right)$ is bounded. A new application of (11), ensures now that $u_{n}\left(t_{n}, x\right)$ is bounded in $H_{0}^{1}(\Omega)$. The sequence $\widehat{u}_{n}\left(t_{n}, x\right)=\alpha_{n} u_{n}\left(t_{n}, x\right)$, where $\alpha_{n}=\sigma /\left\|u_{n}\left(t_{n}, x\right)\right\|_{L^{2}(\Omega)}$, is in $S_{\sigma}$. We have, writing for simplicity $u_{n}, \widehat{u}_{n}$ instead of $u_{n}\left(t_{n}, x\right), \widehat{u}_{n}\left(t_{n}, x\right)$, respectively, for suitable $l_{n}=l_{n}(x) \in(0,1)$, and because of condition (C2),

$$
\begin{aligned}
\left|J\left(\widehat{u}_{n}\right)-J\left(u_{n}\right)\right| \leqslant & \frac{1}{2}\left|\alpha_{n}^{2}-1\right| \int_{\Omega}\left|\nabla u_{n}\right|^{2} d x+\int_{\Omega}\left|W\left(\widehat{u}_{n}\right)-W\left(u_{n}\right)\right| d x \\
= & \frac{1}{2}\left|\alpha_{n}^{2}-1\right| \int_{\Omega}\left|\nabla u_{n}\right|^{2} d x \\
& \quad+\left|\alpha_{n}-1\right| \int_{\Omega}\left|u_{n} W^{\prime}\left(l_{n} u_{n}+\left(1-l_{n}\right) \widehat{u}_{n}\right)\right| d x \\
\leqslant & \frac{1}{2}\left|\alpha_{n}^{2}-1\right| \int_{\Omega}\left|\nabla u_{n}\right|^{2} d x \\
& \quad+\left|\alpha_{n}-1\right| \int_{\Omega} c\left[l_{n}+\left(1-l_{n}\right) \alpha_{n}\right]^{p-1}\left|u_{n}\right|^{p} d x \\
\rightarrow & 0,
\end{aligned}
$$

since in the right hand side of the last inequality, the two summands are products of a zero sequence by a bounded one. Thus, $J\left(\widehat{u}_{n}\right)-J\left(u_{n}\right) \rightarrow 0$. We return now to 


$$
\left\|\psi_{n}(0, x)-\widetilde{w}_{n}\right\|_{H_{0}^{1}(\Omega)} \rightarrow 0
$$

which, as a result of the triangle inequality combined with the boundedness of $\left\|\psi_{n}(0, x)\right\|_{H_{0}^{1}(\Omega)}+\left\|w_{n}(x)\right\|_{H_{0}^{1}(\Omega)}$, readily gives

$$
\left\|\psi_{n}(0, x)\right\|_{H_{0}^{1}(\Omega)}^{2}-\left\|w_{n}(x)\right\|_{H_{0}^{1}(\Omega)}^{2} \rightarrow 0,
$$

that is,

$$
\int_{\Omega}\left[\left|\nabla u_{n}(0, x)\right|^{2}+u_{n}^{2}(0, x)\left|\nabla s_{n}(0, x)\right|^{2}-\left|\nabla w_{n}(x)\right|^{2}\right] d x \rightarrow 0 .
$$

We claim that

$$
\int_{\Omega} u_{n}^{2}(0, x)\left|\nabla s_{n}(0, x)\right|^{2} d x \rightarrow 0
$$

If not so, up to a subsequence,

$$
\int_{\Omega}\left[\left|\nabla u_{n}(0, x)\right|^{2}-\left|\nabla w_{n}(x)\right|^{2}\right] d x \rightarrow k<0 .
$$

Combining $L^{1}$ convergence of $u_{n}(0, x)-w_{n}(x)$ to 0 with condition (C2), we have

$$
\int_{\Omega}\left[W\left(u_{n}(0, x)\right)-W\left(w_{n}(x)\right)\right] d x \rightarrow 0 .
$$

Now (17) and (18) give

$$
J\left(u_{n}(0, x)\right)-J\left(w_{n}(x)\right) \rightarrow \frac{k}{2}<0 .
$$

However, as we have shown above, $J\left(\widehat{u}_{n}(0, x)\right)-J\left(u_{n}(0, x)\right) \rightarrow 0$, thus obtaining

$$
J\left(\widehat{u}_{n}(0, x)\right)-J\left(w_{n}(x)\right) \rightarrow \frac{k}{2}<0,
$$

an absurdity, since $\widehat{u}_{n}(0, x) \in S_{\sigma}$ and $w_{n}(x)$ is a $S_{\sigma}$ minimizer of $J$. Thus (16) holds, and because of (15) and (18), we get

$$
J\left(u_{n}(0, x)\right) \rightarrow \mu .
$$

Thus, $E\left(\psi_{n}(0, x)\right)-E\left(\widetilde{w}_{n}\right) \rightarrow 0$, and by (14) we have

$$
E\left(\psi_{n}\left(t_{n}, x\right)\right)-E\left(\widetilde{w}_{n}\right) \rightarrow 0 .
$$


From (19) we obtain

$$
\int_{\Omega} u_{n}^{2}\left(t_{n}, x\right)\left|\nabla s_{n}\left(t_{n}, x\right)\right|^{2} d x \rightarrow 0 .
$$

To see this, let us assume that this is not the case. Then, up to a subsequence,

$$
\int_{\Omega} u_{n}^{2}\left(t_{n}, x\right)\left|\nabla s_{n}\left(t_{n}, x\right)\right|^{2} d x \rightarrow \rho>0
$$

Then (19) implies that $J\left(u_{n}\left(t_{n}, x\right)\right)-J\left(w_{n}(x)\right) \rightarrow-\rho<0$. Since $J\left(\widehat{u}_{n}\left(t_{n}, x\right)\right)-$ $J\left(u_{n}\left(t_{n}, x\right)\right) \rightarrow 0$, we obtain $J\left(\widehat{u}_{n}\left(t_{n}, x\right)\right)-J\left(w_{n}(x)\right) \rightarrow-\rho<0$, which is absurd, for the same reason as above. That is, (20) holds, resulting to $J\left(u_{n}\right)=J\left(u_{n}\left(t_{n}, x\right)\right) \rightarrow$ $\mu$, thus implying

$$
J\left(\widehat{u}_{n}\left(t_{n}, x\right)\right) \rightarrow \mu
$$

In other words, we may consider $u_{n}=u_{n}\left(t_{n}, x\right)$ as being a minimizing sequence in $S_{\sigma}$ for $J(u)$. As such, by the previous discussion, $u_{n}\left(t_{n}, x\right) \rightarrow u^{\prime}(x)$, with $u^{\prime}(x) \in S_{\sigma}$, being a minimizer of $J(u)$. Now (20) ensures that $\left\|\psi_{n}\left(t_{n}, x\right)\right\|_{H_{0}^{1}(\Omega)} \rightarrow\left\|u^{\prime}(x)\right\|_{H_{0}^{1}(\Omega)}$, and by uniform convexity of the space we finally obtain that $\psi_{n}\left(t_{n}, x\right) \rightarrow u^{\prime}(x)$, thus proving orbital stability of the stationary solution.

Remark 2.3 As we claimed before, once $\psi(0, x)$ is sufficiently close to $\Gamma, \psi(t, x)$, $t \geqslant 0$, are all close to $\Gamma$. This implies that the modulus $|\psi(t, x)|$ is, for any $t \geqslant 0$, close to a (possibly different) ground state of the NLSE. This consequence is the key tool for proving two main results of the next section, Lemmas 3.8 and 3.9. Thus, the orbital stability in this context becomes a localization result for the moduli $|\psi(t, x)|$.

We summarize the existence and stability results in the following statement.

Proposition 2.4 The problem

$$
\begin{array}{rlrl}
i h \frac{\partial \psi}{\partial t} & =-\frac{h^{2}}{2} \Delta \psi+\frac{1}{2 h^{\alpha}} W^{\prime}(|\psi|) \frac{\psi}{|\psi|}, & (t, x) \in \mathbb{R}_{0}^{+} \times \Omega, \\
\psi(0, x) & =\phi_{h}(x), & x \in \Omega, & \\
\psi(t, x) & =0, & (t, x) \in \mathbb{R}_{0}^{+} \times \partial \Omega,
\end{array}
$$

where $h>0$ and $\phi_{h}(x)$ is a suitable initial datum, admits a stationary solution $\psi(t, x)$ of the form $u_{h}(x) e^{-i k t}$. More concretely, $u_{h}(x)$ is obtained as a solution of the eigenvalue problem

$$
\begin{aligned}
-\frac{h}{2} \Delta u+\frac{1}{2 h^{\alpha+1}} W^{\prime}(u) & =k u \quad \text { in } \Omega, \\
u & \equiv 0 \quad \text { on } \partial \Omega,
\end{aligned}
$$

for suitable $k$. In addition, $\psi(t, x)$ is stable in the sense of $(7)$. 
We give next the definition of a solitary wave with respect to a bounded domain $\Omega$. To this end, we have to define first the notion of the barycenter of a family of states $\psi_{t}(x), t \geqslant 0$, whose members are obtained by the "time" evolution of initial state $\psi_{0}(x)$, in the frame of proper phase space $X \subset L^{2}(\Omega, \mathbb{C})$.

Definition 2.5 For $\psi_{t}(x), t \geqslant 0$, as above, its barycenter $q(t)$ is defined by the relation

$$
q(t)=\frac{\int_{\Omega} x\left|\psi_{t}(x)\right|^{2} d x}{\int_{\Omega}\left|\psi_{t}(x)\right|^{2} d x} .
$$

Remark 2.6 An analogous definition of the barycenter is given in [3], under the condition that it makes sense. In our case, the definition of $q(t)$ always makes sense, because $\Omega$ is bounded.

Remark 2.7 Notice that $q(t)$ does not belong to $\Omega$ necessarily, unless $\Omega$ has specific geometric features. For instance, convexity of $\Omega$ would ensure that $q(t) \in \Omega$ for all $t \geqslant 0$.

Definition 2.8 The state $\psi \equiv \psi_{0}(x)$ in the phase space $X \subset L^{2}(\Omega, \mathbb{C})$, is called a "solitary wave" in the frame of a dynamical system $U_{t} \psi \equiv \psi_{t}(x), t \geqslant 0$, where $U: \mathbb{R}_{0}^{+} \times X \rightarrow X$ is an evolution map, if: given $\varepsilon>0$, we may find $k(\varepsilon)>0$ such that for each $t \geqslant 0$, there exists a neighborhood $V_{\varepsilon, t}$ of $q(t)$ with meas $\left[\Omega-\left(V_{\varepsilon, t} \cap \Omega\right)\right] \geqslant$ $k(\varepsilon)$, and

$$
\int_{\Omega}\left|\psi_{t}(x)\right|^{2} d x-\int_{V_{\varepsilon, t} \cap \Omega}\left|\psi_{t}(x)\right|^{2} d x<\varepsilon .
$$

Remark 2.9 It is easy to see that the stationary solution of (21) is a solitary wave in the above sense: The barycenter in this case is fixed for all $t$, and one needs to blow up suitably a given neighborhood of it in order to meet the requirements of the above definition.

\section{$3 L^{2}$ localization for $V \neq 0$}

For the rest of exposition, we assume without loss of generality that $0 \in \Omega$. We also restrict $p$ in condition (C2) so that $2<p<2+4 / N$, and we impose on $W$ the following additional condition:

(C3) there exists $s_{0}$ such that $W\left(s_{0}\right)<0$,

and on $V$ the following one:

(C4) $V(x) \in C^{0}(\bar{\Omega})$ is nonnegative.

Remark 3.1 With these restrictions and additional conditions on the nonlinearity and potential, problem (1) is globally well-posed (see [11, Theorems 3.3.1 and 3.4.1]) with the energy and the mass remaining constant in time. Commenting especially on 
condition ( $\mathrm{C} 3$ ), we notice that in the opposite case, there would exist no nontrivial stationary solution for the related $\mathbb{R}^{N}$ problem, undermining thus the basic tool we will use in order to prove the main localization result. In the previous section, where we had posed weaker conditions on $\Omega, W$, and $p$, we were led to nontrivial stationary solution without any particular comment on $\lambda$. It is trivial to see that for $W$ having positive lower bound, $\lambda$ is positive, too. Under the additional conditions imposed above on $\Omega, W$, and $p$, we can prove, as we will see soon, that we may obtain a nontrivial solution of (3) sitting in $S_{\sigma}$ for suitable $\sigma$, with $\lambda=\lambda(\sigma)<0$, provided that $\Omega$ contains a suitably big open ball $B(0, r(\sigma))$ centered at 0 with radius $r(\sigma)$.

\subsection{Rescalings}

We set $\beta=1+\alpha / 2$. For $h<1$, we define the inflated domain $\Omega_{h}=\left\{x \in \mathbb{R}^{N}\right.$ : $h^{\beta} x \in \Omega$ \}. If $v$ is an $H_{0}^{1}\left(\Omega_{h}\right)$ solution of the stationary problem

$$
\begin{aligned}
-\Delta u+W^{\prime}(u) & =\omega u & & \text { in } \Omega_{h}, \\
u & \equiv 0 & & \text { on } \partial \Omega_{h},
\end{aligned}
$$

then $v_{h}(x)=v\left(x / h^{\beta}\right)$ is an $H_{0}^{1}(\Omega)$ solution of the stationary problem

$$
\begin{aligned}
-h \Delta u+\frac{1}{h^{\alpha+1}} W^{\prime}(u) & =\frac{\omega}{h^{\alpha+1}} u & & \text { in } \quad \Omega, \\
u & \equiv 0 & & \text { on } \quad \partial \Omega .
\end{aligned}
$$

Furthermore, we define the functionals

$$
\begin{aligned}
C_{h}(u) & =\frac{1}{h^{N \beta}} \int_{\Omega} u^{2}(x) d x, & J_{h}(u) & =\frac{1}{h^{N \beta}} \int_{\Omega}\left[\frac{h^{2}}{2}|\nabla u|^{2}+W_{h}(u)\right] d x, \\
C_{\Omega_{h}}(u) & =\int_{\Omega_{h}} u^{2}(x) d x, & J_{\Omega_{h}}(u) & =\int_{\Omega_{h}}\left[\frac{1}{2}|\nabla u|^{2}+W(u)\right] d x,
\end{aligned}
$$

where $W_{h}(u)=W(u) / h^{\alpha}$. We have the following identities:

$$
J_{h}\left(v_{h}\right)=h^{-\alpha} J_{\Omega_{h}}(v), \quad C_{h}\left(v_{h}\right)=C_{\Omega_{h}}(v) .
$$

We next define $m(h, \Omega)=\inf _{C_{h}=1} J_{h}$.

Lemma 3.2 For $\Omega_{1}, \Omega_{2}$ two bounded domains as described above, with $\Omega_{1} \subset \Omega_{2}$, $m\left(h, \Omega_{2}\right) \leqslant m\left(h, \Omega_{1}\right)$.

Proof It is straightforward, since $H_{0}^{1}\left(\Omega_{1}\right) \subset H_{0}^{1}\left(\Omega_{2}\right)$ by extending a function in $H_{0}^{1}\left(\Omega_{1}\right)$ into a function in $H_{0}^{1}\left(\Omega_{2}\right)$ by zero on $\Omega_{2} \backslash \Omega_{1}$.

The following relation is an easy consequence of rescaling. 
Lemma $3.3 m(h, \Omega)=h^{-\alpha} m\left(1, \Omega_{h}\right)$, where $m\left(1, \Omega_{h}\right)=\inf _{C_{\Omega_{h}}=1} J_{\Omega_{h}}$.

Notice that the conditions satisfied by $p, W$ correspond to the prerequisites for the existence result given in [4] to hold. Namely

Lemma 3.4 There exists some $\bar{\sigma}>0$ such that for all $\sigma>\bar{\sigma}$ a positive minimizer $u_{\sigma} \in H^{1}\left(\mathbb{R}^{N}\right)$ exists for $J(u)$ over all $u \in H^{1}\left(\mathbb{R}^{N}\right),\|u\|_{L^{2}\left(\mathbb{R}^{N}\right)}=\sigma$. In fact, $\left\|u_{\sigma}\right\|_{L^{2}\left(\mathbb{R}^{N}\right)}=\sigma, J\left(u_{\sigma}\right)<0$, and $u_{\sigma}$ is a solution of the $\mathbb{R}^{N}$ version of (3) with $\lambda<0$.

We consider an increasing sequence $\left\{r_{n}\right\}$, with $r_{n} \rightarrow \infty$. Let

$$
m_{\sigma}\left(B\left(0, r_{n}\right)\right)=\inf _{\|w\|_{L^{2}\left(B\left(0, r_{n}\right)\right)}=\sigma} J(w) .
$$

Trivially, as Lemma 3.2 indicates, we see that

$$
\begin{aligned}
m_{\sigma}\left(B\left(0, r_{1}\right)\right) \geqslant m_{\sigma}\left(B\left(0, r_{2}\right)\right) \geqslant \cdots & \geqslant m_{\sigma}\left(\mathbb{R}^{N}\right) \\
& =\inf _{\|w\|_{L^{2}\left(\mathbb{R}^{N}\right)}=\sigma} J(w)>-\infty .
\end{aligned}
$$

Lemma $3.5 \lim _{n \rightarrow \infty} m_{\sigma}\left(B\left(0, r_{n}\right)\right)=m_{\sigma}\left(\mathbb{R}^{N}\right)$.

Proof By Lemma 3.4, $m_{\sigma}\left(\mathbb{R}^{N}\right)$ is attained by some $\bar{u} \in H^{1}\left(\mathbb{R}^{N}\right)$, with $\|\bar{u}\|_{L^{2}\left(\mathbb{R}^{N}\right)}=$ $\sigma$. Actually $\bar{u}$ is radial, [12, Theorem II.1, Remark II.3].

For each $n$, we may choose a $C^{\infty}\left(\mathbb{R}^{N}\right)$ real function $\chi_{n}$ satisfying

$$
\chi_{n} \equiv\left\{\begin{array}{ll}
1 & \text { if } \quad|x| \leqslant \frac{r_{n}}{2}, \\
0 & \text { if }|x| \geqslant r_{n},
\end{array} \quad\left|\nabla \chi_{n}\right| \leqslant \frac{4}{r_{n}} .\right.
$$

We define $w_{n}=\chi_{n} \bar{u}$. For suitable $t_{n}>0$, we have $\left\|t_{n} w_{n}\right\|_{L^{2}\left(\mathbb{R}^{N}\right)}=\left\|t_{n} w_{n}\right\|_{L^{2}\left(B\left(0, r_{n}\right)\right)}$ $=\sigma$. Setting $u_{n}=t_{n} w_{n},(25)$ yields

$$
J\left(u_{n}\right) \geqslant m_{\sigma}\left(B\left(0, r_{n}\right)\right) \geqslant m_{\sigma}\left(\mathbb{R}^{N}\right) .
$$

We want to prove $\lim _{n \rightarrow \infty} J\left(u_{n}\right)=m_{\sigma}\left(\mathbb{R}^{N}\right)$ that will finish the proof. We have $w_{n} \rightarrow \bar{u}$ in $L^{2}\left(\mathbb{R}^{N}\right)$. Thus $t_{n} \rightarrow 1$, and $u_{n} \rightarrow \bar{u}$ in $L^{2}\left(\mathbb{R}^{N}\right)$. We also have

$$
\begin{aligned}
\int_{\mathbb{R}^{N}}\left|\nabla w_{n}-\nabla \bar{u}\right|^{2} d x & =\int_{\mathbb{R}^{N}}\left|\left(\nabla \chi_{n}\right) \bar{u}+\left(\chi_{n}-1\right) \nabla \bar{u}\right|^{2} d x \\
& \leqslant 2 \int_{\mathbb{R}^{N}}\left|\left(\nabla \chi_{n}\right) \bar{u}\right|^{2} d x+2 \int_{\mathbb{R}^{N}}\left|\left(\chi_{n}-1\right) \nabla \bar{u}\right|^{2} d x \\
& \leqslant \frac{32}{r_{n}^{2}} \int_{|x|>r_{n} / 2}|\bar{u}|^{2} d x+2 \int_{|x|>r_{n} / 2}|\nabla \bar{u}|^{2} d x \rightarrow 0
\end{aligned}
$$


as $n \rightarrow \infty$. So $\nabla w_{n} \rightarrow \nabla \bar{u}$ in $L^{2}$ and $\nabla u_{n} \rightarrow \nabla \bar{u}$ in $L^{2}$ too. Thus $u_{n} \rightarrow \bar{u}$ in $H^{1}\left(\mathbb{R}^{N}\right)$. This is combined with the continuity of the Nemytskii operator

$$
W: L^{t}\left(\mathbb{R}^{N}\right) \rightarrow L^{1}\left(\mathbb{R}^{N}\right), \quad 2<t<2^{*},
$$

to ensure that

$$
\int_{\mathbb{R}^{N}} W\left(u_{n}\right) d x \rightarrow \int_{\mathbb{R}^{N}} W(\bar{u}) d x .
$$

Thus $\int_{n \rightarrow \infty} J\left(u_{n}\right)=m_{\sigma}\left(\mathbb{R}^{N}\right)$, and the proof is complete.

Remark 3.6 The above lemma makes clear the final assertion of Remark 3.1: if $\Omega$ contains a suitably big open ball $B(0, r(\sigma))$ with $\bar{\sigma}<\sigma$, then $m_{\sigma}(1, \Omega)$ has to be negative, and so has to be the eigenvalue $\lambda=\lambda(\sigma)$ related to (3).

The next result is a consequence of Lemmas 3.2 and 3.5.

Lemma 3.7 For a sequence of positive numbers $h_{k} \rightarrow 0, k \rightarrow \infty$,

$$
\lim _{k \rightarrow \infty} m_{\sigma}\left(1, \Omega_{h_{k}}\right)=m_{\sigma}\left(\mathbb{R}^{N}\right),
$$

where $m_{\sigma}\left(1, \Omega_{h_{k}}\right)=\inf _{C_{\Omega_{h_{k}}}=\sigma} J_{\Omega_{h_{k}}}$.

\section{2 $L^{2}$ localization}

In order to facilitate exposition, we make a harmless assumption that $\sigma=1$, thus suppressing subindices in all involved infima $m$. We have the following

Lemma 3.8 For any $\varepsilon>0$, there exist $\Delta=\Delta(\varepsilon), h_{0}=h_{0}(\varepsilon)>0$ and $R=R(\varepsilon)>0$ such that, for any $0<h<h_{0}(\varepsilon)$, there is an open ball $B\left(\widehat{q}_{h}, h^{\beta} R\right) \subset \Omega$ so that for any $u \in H_{0}^{1}(\Omega)$ with $C_{h}(u)=1$ and $J_{h}(u)<m(h, \Omega)+\Delta h^{-\alpha}$,

$$
\frac{1}{h^{N \beta}} \int_{\Omega \backslash B\left(\widehat{q}_{h}, h^{\beta} R\right)} u^{2} d x<\varepsilon .
$$

Proof We argue by contradiction. Assuming the contrary, there exists $\varepsilon>0$ such that for any $r>0$ we may find sequences $\Delta_{n}=\Delta_{n}(r), h_{n}=h_{n}(r) \rightarrow 0^{+}$, and a sequence $u_{h_{n}}=u_{h_{n}}(r) \in H_{0}^{1}(\Omega)$ with $C_{h_{n}}\left(u_{h_{n}}\right)=1, J_{h_{n}}\left(u_{h_{n}}\right)<m\left(h_{n}, \Omega\right)+\Delta_{n} h_{n}^{-\alpha}$ such that, for all open balls $B\left(q_{r}, h_{n}^{\beta} r\right) \subset \Omega$,

$$
\int_{\Omega \backslash B\left(q_{r}, h_{n}^{\beta} r\right)} u_{h_{n}}^{2} d x \geqslant \varepsilon h_{n}^{N \beta} .
$$

For each $n$, we now pass to the $\Omega_{h_{n}}$ counterpart of $u_{h_{n}}$, denoted by $u_{n}$, that is, $u_{n}(x)=$ $u_{h_{n}}\left(h_{n}^{\beta} x\right)$. Combining (24) and Lemma 3.3, we have $C_{\Omega_{h_{n}}}\left(u_{n}\right)=1, J_{\Omega_{h_{n}}}\left(u_{n}\right)<$ $m\left(1, \Omega_{h_{n}}\right)+\Delta_{n}$ and 


$$
\int_{\Omega_{h_{n}} \backslash B\left(h_{n}^{-\beta} q_{r}, r\right)} u_{n}^{2} d x \geqslant \varepsilon .
$$

According to [6, Lemma 15], there is $\Delta>0$ and an open ball $B\left(\widehat{q}, R^{\prime}\right)$ in $\mathbb{R}^{N}$ such that, for each $w$ in $H^{1}\left(\mathbb{R}^{N}\right)$, with $\|w\|_{L^{2}\left(\mathbb{R}^{N}\right)}=1, J(w)<m\left(\mathbb{R}^{N}\right)+\Delta$,

$$
\int_{\mathbb{R}^{N} \backslash B\left(\widehat{q}, R^{\prime}\right)} w^{2} d x<\varepsilon
$$

Take now $\Delta_{n}\left(R^{\prime}\right), h_{n}\left(R^{\prime}\right)$ as above. Because of Lemma 3.7 for $n$ big enough, we may ensure that $B\left(\widehat{q}, R^{\prime}\right) \subset \Omega_{h_{n}\left(R^{\prime}\right)}$ and $m\left(1, \Omega_{h_{n}}\right)+\Delta_{n}\left(R^{\prime}\right)<m\left(\mathbb{R}^{N}\right)+\Delta$. Extending $u_{n}$ by 0 outside $\Omega_{h_{n}\left(R^{\prime}\right)}$, we obtain an $H^{1}\left(\mathbb{R}^{N}\right)$ function meeting the requirements of $[6$, Lemma 15], thus

$$
\int_{\Omega_{h_{n}\left(R^{\prime}\right) \backslash B\left(\widehat{q}, R^{\prime}\right)}} u_{n}^{2} d x=\int_{\mathbb{R}^{N} \backslash B\left(\widehat{q}, R^{\prime}\right)} u_{n}^{2} d x<\varepsilon,
$$

that contradicts (26).

Lemma 3.8 makes obvious the following

Lemma 3.9 For any $\varepsilon>0$, there exist $\Delta=\Delta(\varepsilon), h_{0}=h_{0}(\varepsilon)>0$ and $R=R(\varepsilon)>0$ such that, for any $0<h<h_{0}(\varepsilon)$, there is an open ball $B\left(\widehat{q}_{h}, h^{\beta} R\right) \subset \Omega$ so that for a solution $\psi(t, x)$ of $(1)$ with $C_{h}(|\psi(t, x)|)=1$ and $J_{h}(|\psi(t, x)|)<m(h, \Omega)+\Delta h^{-\alpha}$, for each $t \in \mathbb{R}_{0}^{+}$,

$$
\frac{1}{h^{N \beta}} \int_{\Omega \backslash B\left(\widehat{q}_{h}, h^{\beta} R\right)}|\psi(t, x)|^{2} d x<\varepsilon .
$$

The correlation of the solutions of equations (22) and (23), combined with (24), ensure the existence of "ground state" solutions of (23), that is, solutions that are minimizers of $J_{h}(u)$ with $u$ satisfying $C_{h}(u)=1$.

We define next, the following set of admissible initial data, for given $K, h>0$ :

$$
B_{h}^{K}=\left\{\begin{array}{l}
\psi(0, x)=u_{h}(0, x) e^{i s_{h}(0, x) / h} \text { with } u_{h}(0, x)=(U+w)(x), \\
U \text { is a ground state solution of }(23), \\
w \in H_{0}^{1}(\Omega) \text { is such that } C_{h}(U+w)=1,\|w\|_{H_{0}^{1}(\Omega)}<K h^{\alpha}, \\
\left\|\nabla s_{h}(0, x)\right\|_{L^{\infty}} \leqslant K h^{N \beta / 2}, \\
\int_{\Omega} V(x) u_{h}^{2}(0, x) d x \leqslant K h^{N \beta}
\end{array}\right\} .
$$

We prove next the basic localization result.

Proposition 3.10 Given $\varepsilon>0$, there exists $h_{0}=h_{0}(\varepsilon)>0$ and $R=R(\varepsilon)>0$ such that, for any $0<h<h_{0}(\varepsilon)$, there is an open ball $B\left(\widehat{q}_{h}, h^{\beta} R\right) \subset \Omega$ so that for a solution $\psi(t, x)$ of $(1)$ with $C_{h}(|\psi(t, x)|)=1$, and with initial data $\psi(0, x) \in B_{h}^{K}$, where $K$ is a positive fixed number, 


$$
\frac{1}{h^{N \beta}} \int_{\Omega \backslash B\left(\widehat{q}_{h}, h^{\beta} R\right)}|\psi(t, x)|^{2} d x<\varepsilon
$$

for any $t \in \mathbb{R}_{0}^{+}$.

Proof Because of conservation of energy, we have

$$
\begin{aligned}
E(\psi(t, x)) & =E(\psi(0, x)) \\
& =h^{N \beta} J_{h}\left(u_{h}(0, x)\right)+\int_{\Omega} u_{h}^{2}(0, x)\left[\frac{\left|\nabla s_{h}(0, x)\right|^{2}}{2}+V(x)\right] d x \\
& \leqslant h^{N \beta} J_{h}\left(u_{h}(0, x)\right)+\frac{K^{2}}{2} h^{N \beta}+K h^{N \beta} \\
& =h^{N \beta} J_{h}\left(u_{h}(0, x)\right)+C h^{N \beta} \\
& =h^{N \beta} J_{h}(U+w)+C h^{N \beta} \\
& \leqslant h^{N \beta}\left(m(h, \Omega)+C^{\prime} K h^{\alpha}+C\right) \\
& =h^{N \beta}\left[m(h, \Omega)+h^{-\alpha}\left(h^{2 \alpha} C^{\prime} K+C h^{\alpha}\right)\right],
\end{aligned}
$$

since a mean value theorem application ensures that $J_{h}(U+w) \leqslant m(h, \Omega)+C^{\prime} K h^{\alpha}$ for suitably small $h$. More precisely, since $J_{h}$ is $C^{1}$, we may find some $\eta \in(0,1)$ so that

$$
\begin{aligned}
J_{h}(U+w)-J_{h}(U) & =J_{h}^{\prime}(U+\eta w)[w] \\
& =\int_{\Omega} \nabla(U+\eta w) \nabla w d x+\int_{\Omega} W^{\prime}(U+\eta w) w d x
\end{aligned}
$$

We have

$$
\begin{aligned}
\left|\int_{\Omega} \nabla(U+\eta w) \nabla w d x\right| & \leqslant\|\nabla U\|_{L^{2}(\Omega)}\|\nabla w\|_{L^{2}(\Omega)}+\|\nabla w\|_{L^{2}(\Omega)}^{2} \\
& \leqslant C_{1}\|w\|_{H_{0}^{1}(\Omega)},
\end{aligned}
$$

since $\|w\|_{H_{0}^{1}(\Omega)} \leqslant 1$. Because of condition $(\mathrm{C} 2)$,

$$
\left|\int_{\Omega} W^{\prime}(U+\eta w) w d x\right| \leqslant C_{2}\|w\|_{L^{p}(\Omega)} \leqslant C_{3}\|w\|_{H_{0}^{1}(\Omega)} .
$$

Combining (27) and (28), we obtain the desired inequality. Thus

$$
\begin{aligned}
J_{h}\left(u_{h}(t, x)\right) & =h^{-N \beta}\left\{E(\psi(t, x))-\int_{\Omega} u_{h}^{2}(t, x)\left[\frac{\left|\nabla s_{h}(t, x)\right|^{2}}{2}+V(x)\right] d x\right\} \\
& \leqslant\left[m(h, \Omega)+h^{-\alpha}\left(h^{2 \alpha} C^{\prime} K+C h^{\alpha}\right)\right]
\end{aligned}
$$

since $V(x) \geqslant 0$. We use now Lemma 3.9, by choosing $h_{0}$ small enough in order to ensure $h_{0}^{2 \alpha} C^{\prime} K+C h_{0}^{\alpha} \leqslant \Delta(\varepsilon)$, and the result follows. 
Corollary 3.11 Given $\varepsilon>0$, if $q(t)$ is the barycenter of a solution $\psi(t, x)$ of (1) with $C_{h}(|\psi(t, x)|)=1$, and with initial data $\psi(0, x) \in B_{h}^{K}$, where $K$ is a positive fixed number, then for any $t \in \mathbb{R}_{0}^{+}, q(t) \in B\left(\widehat{q}_{h}, d \varepsilon+h^{\beta} R\right)$, where $h$ and $R$ are as in Proposition 3.10, and $d=\operatorname{diam} \Omega$.

Proof One has for any $t \in \mathbb{R}_{0}^{+}$,

$$
\begin{aligned}
\left|\widehat{q}_{h}-q(t)\right| & =\left|\frac{\int_{\Omega}\left(\widehat{q}_{h}-x\right)|\psi(t, x)|^{2} d x}{\int_{\Omega}|\psi(t, x)|^{2} d x}\right|=\left.h^{-N \beta}\left|\int_{\Omega}\left(\widehat{q}_{h}-x\right)\right| \psi(t, x)\right|^{2} d x \mid \\
& \leqslant h^{-N \beta} \int_{\Omega}\left|\widehat{q}_{h}-x\right||\psi(t, x)|^{2} d x \\
& =h^{-N \beta}\left[\int_{\Omega \backslash B\left(\widehat{q}_{h}, h^{\beta} R\right)}\left|\widehat{q}_{h}-x\right||\psi(t, x)|^{2} d x\right. \\
& \left.+\int_{B\left(\widehat{q}_{h}, h^{\beta} R\right)}\left|\widehat{q}_{h}-x\right||\psi(t, x)|^{2} d x\right] \\
& <h^{-N \beta}\left[d h^{N \beta} \varepsilon+h^{\beta} R h^{N \beta}\right]=d \varepsilon+h^{\beta} R,
\end{aligned}
$$

thus proving the claim.

Remark 3.12 The above corollary may be interpreted as follows: if we choose a pretty small $\varepsilon>0$ and make a consequent choice of small $h$, then $B\left(\widehat{q}_{h}, h^{\beta} R\right) \subset A_{h}=$ $B\left(\widehat{q}_{h}, d \varepsilon+h^{\beta} R\right) \cap \Omega$, with meas $\left(\Omega-A_{h}\right)>0$. In other words, we have found a neighborhood of $q(t)$, for any $t \in \mathbb{R}_{0}^{+}$, as in Definition 2.8 , in the sense that a solution $\psi(t, x)$ of (1) with a perturbed initial state, meeting the requirements of Proposition 3.10, concentrates basically on this neighborhood of $q(t)$, exhibiting a behavior quite similar to a solitary wave.

\section{$4 H^{1}$ localization for $V \neq 0$}

\subsection{Case $\Omega=\mathbb{R}^{N}$}

We start with the following assumption: the problem

$$
\begin{aligned}
i h \frac{\partial \psi}{\partial t} & =-\frac{h^{2}}{2} \Delta \psi+\frac{1}{2 h^{\alpha}} W^{\prime}(|\psi|) \frac{\psi}{|\psi|}+V(x) \psi, \\
\psi(0, x) & =\phi_{h}(x), \\
|\psi(t, x)| & \rightarrow 0 \quad \text { as } \quad|x| \rightarrow \infty,
\end{aligned}
$$

considered as an $\mathbb{R}^{N}$ analogue of (1) admits a unique solution

$$
\psi \in C^{0}\left(\mathbb{R}, H^{2}\left(\mathbb{R}^{N}\right)\right) \cap C^{1}\left(\mathbb{R}, L^{2}\left(\mathbb{R}^{N}\right)\right)
$$

(see $[11,17]$ or [18] for sufficient conditions). We also impose on $W, V$ further conditions, namely: 
(C5) $W$ is $C^{3}$, with $\left|W^{\prime \prime}\right| \leqslant c|s|^{p-2}$ for some $c \geqslant 0,2<p<2+4 / N$.

(C6) $V: \mathbb{R}^{N} \rightarrow \mathbb{R}$ is a $C^{0}$ nonnegative function.

In order to proceed, we will need the following lemma.

Lemma 4.1 For every $\varepsilon>0$, there exists $R=R(\varepsilon)>0$ such that, for every ground state $U$, there exists $q(U) \in \mathbb{R}^{N}$ such that

$$
\int_{\mathbb{R}^{N} \backslash B(q(U), R)}\left(|\nabla U|^{2}+U^{2}\right) d x<\varepsilon .
$$

Proof If we assume the contrary, then we may find $\varepsilon>0$ such that we may have a sequence of pairs $\left(R_{n}>0, U_{n}\right.$ ground state) so that for each $q \in \mathbb{R}^{N}$

$$
\int_{\mathbb{R}^{N} \backslash B\left(q(U), R_{n}\right)}\left(\left|\nabla U_{n}\right|^{2}+U_{n}^{2}\right) d x \geqslant \varepsilon,
$$

thus obtaining

$$
\inf _{q \in \mathbb{R}^{N}} \int_{\mathbb{R}^{N} \backslash B\left(q(U), R_{n}\right)}\left(\left|\nabla U_{n}\right|^{2}+U_{n}^{2}\right) d x \geqslant \varepsilon .
$$

Then $\left\{U_{n}\right\}_{n}$ is a minimizing sequence and by concentration compactness we know that $\left\{U_{n}\right\}_{n}$ is relatively compact up to a translation by $\left\{q_{n}\right\}_{n} \in \mathbb{R}^{N}$. Thus there exists a ground state $U$ with $U_{n}\left(\cdot-q_{n}\right) \rightarrow U$ in $H^{1}\left(\mathbb{R}^{N}\right)$, and

$$
\begin{aligned}
\inf _{q \in \mathbb{R}^{N}} \int_{\mathbb{R}^{N} \backslash B\left(q(U), R_{n}\right)} & \left(\left|\nabla U_{n}\right|^{2}+U_{n}^{2}\right) d x \leqslant \int_{\mathbb{R}^{N} \backslash B\left(-q_{n}, R_{n}\right)}\left(\left|\nabla U_{n}\right|^{2}+U_{n}^{2}\right) d x \\
= & \int_{\mathbb{R}^{N} \backslash B\left(0, R_{n}\right)}\left(\left|\nabla U_{n}\right|^{2}+U_{n}^{2}\right)\left(x-q_{n}\right) d x \\
= & \int_{\mathbb{R}^{N} \backslash B\left(0, R_{n}\right)}\left(|\nabla U|^{2}+U^{2}\right) d x+\mathrm{o}_{n}(1)=\mathrm{o}_{n}(1),
\end{aligned}
$$

contradicting (30).

Lemma 4.2 For every $\varepsilon>0$, there exist $\widehat{R}=\widehat{R}(\varepsilon)>0$ and $\Delta=\Delta(\varepsilon)>0$ such that, for any $u \in J^{m+\Delta} \cap S_{\sigma}$, we can find a point $\widehat{q}=\widehat{q}(u) \in \mathbb{R}^{N}$ such that

$$
\frac{\int_{\mathbb{R}^{N} \backslash B(\widehat{q}, \widehat{R})}|\nabla u|^{2}(x) d x}{\int_{\mathbb{R}^{N}}|\nabla u|^{2}(x) d x}<\varepsilon,
$$

where $m=m\left(\mathbb{R}^{N}\right)\left(\right.$ see Lemma 3.8), $J^{m+\Delta}=\left\{u \in H^{1}\left(\mathbb{R}^{N}\right): J(u)<m+\Delta\right\}$.

Proof Exploiting (50) in [6], we obtain a point $\widehat{q}=\mathbb{R}^{N}$ and a radial ground state solution $U$ such that

$$
u(x)=U(x-\widehat{q})+w \quad \text { with } \quad\|w\|_{H^{1}\left(\mathbb{R}^{N}\right)} \leqslant C \varepsilon
$$


where $C$ is a constant not depending on $U$. According to the previous lemma, we may find $R>0$ and a point $q=q(U)$ such that

$$
\int_{\mathbb{R}^{N} \backslash B(q, R)}\left(|\nabla U|^{2}+U^{2}\right) d x<\frac{\sigma^{2} C \varepsilon}{c_{1}^{2}},
$$

where $c_{1}$ is the Sobolev constant related to the embedding $H^{1}\left(\mathbb{R}^{N}\right) \rightarrow L^{2}\left(\mathbb{R}^{N}\right)$. If we choose $\widehat{R}$ big enough then $B(q, R) \subset B(0, \widehat{R})$, resulting to

$$
\int_{\mathbb{R}^{N} \backslash B(0, \widehat{R})}\left(|\nabla U|^{2}+U^{2}\right) d x<\frac{\sigma^{2} C \varepsilon}{c_{1}^{2}} .
$$

We have

$$
\frac{\int_{\mathbb{R}^{N} \backslash B(0, \widehat{R})}|\nabla U|^{2}(x) d x}{\int_{\mathbb{R}^{N}}|\nabla U|^{2}(x) d x}<\frac{c_{1}^{2} \int_{\mathbb{R}^{N} \backslash B(0, \widehat{R})}\left(|\nabla U|^{2}+U^{2}\right) d x}{\int_{\mathbb{R}^{N}} U^{2} d x}<C \varepsilon .
$$

Now

$$
\begin{aligned}
\int_{\mathbb{R}^{N} \backslash B(\widehat{q}, \widehat{R})}|\nabla u|^{2}(x) d x \\
<\int_{\mathbb{R}^{N} \backslash B(\widehat{q}, \widehat{R})}|\nabla U|^{2}(x-\widehat{q}) d x+\int_{\mathbb{R}^{N} \backslash B(\widehat{q}, \widehat{R})}\left(|\nabla w|^{2}+2 w U\right) d x \\
=\int_{\mathbb{R}^{N} \backslash B(0, \widehat{R})}|\nabla U|^{2}(x) d x+\int_{\mathbb{R}^{N} \backslash B(\widehat{q}, \widehat{R})}\left(|\nabla w|^{2}+2 \nabla w \nabla U\right) d x .
\end{aligned}
$$

By (31), (33) and (34), we get the claim. One should notice that $\widehat{R}$ does not depend on $u, U$.

Our main objective in this subsection is to prove an $H^{1}$ modular localization result of the solution of (29) with suitable initial data; more precisely, we prove that, for fixed $t \in \mathbb{R}_{0}^{+}$, this solution is a function on $\mathbb{R}^{N}$ with one peak localized in a ball with its center depending on $t$ whereas its radius not. To this end, it is sufficient to assume that (29) admits global solutions $\psi(t, x) \in C\left(\mathbb{R}, H^{1}\left(\mathbb{R}^{N}\right)\right)$ satisfying the conservation of the energy and of the $L^{2}$ norm.

Given $K>0, h>0$, we define the following set of admissible data:

$$
B_{K, h}=\left\{\begin{array}{l}
\psi(0, x)=u_{h}(0, x) e^{i s_{h}(0, x) / h} \text { with } u_{h}(0, x)=(U+w)\left(\frac{x-q}{h^{\beta}}\right), \\
q \in \mathbb{R}^{N}, U \text { is a ground state solution, } \\
w \in H^{1}\left(\mathbb{R}^{N}\right) \text { such that }\|U+w\|_{L^{2}}=\|U\|_{L^{2}}=\sigma,\|w\|_{H^{1}}<K h^{\alpha}, \\
\left\|\nabla s_{h}(0, x)\right\|_{L^{\infty}} \leqslant K \text { for all } h, \\
\int_{\mathbb{R}^{N}} V(x) u_{h}^{2}(0, x) d x \leqslant K h^{N \beta-2 \alpha}
\end{array}\right\} .
$$


We next study the rescaling properties of the internal energy

$$
\widetilde{J}_{h}(u)=\int_{\mathbb{R}^{N}}\left(\frac{h^{2}}{2}|\nabla u|^{2}+\frac{1}{h^{\alpha}} W(u)\right) d x,
$$

and of the $L^{2}$ norm of a function $u(x)$ having the form $u(x)=v\left(x / h^{\beta}\right)$, with $\beta=1+\alpha / 2$. We have

$$
\|u\|_{L^{2}}^{2}=\int_{\mathbb{R}^{N}} v^{2}\left(\frac{x}{h^{\beta}}\right) d x=h^{N \beta} \int_{\mathbb{R}^{N}} v^{2}(\xi) d \xi=h^{N \beta}\|v\|_{L^{2}}^{2},
$$

and

$$
\begin{aligned}
\widetilde{J}_{h}(u) & =\int_{\mathbb{R}^{N}}\left[\frac{h^{2}}{2}\left|\nabla_{x} v\left(\frac{x}{h^{\beta}}\right)\right|^{2}+\frac{1}{h^{\alpha}} W\left(v\left(\frac{x}{h^{\beta}}\right)\right)\right] d x \\
& =\int_{\mathbb{R}^{N}}\left[\frac{h^{(N-2) \beta+2}}{2}\left|\nabla_{\xi} v(\xi)\right|^{2}+h^{N \beta-\alpha} W(v(\xi))\right] d \xi \\
& =h^{N \beta-\alpha} \int_{\mathbb{R}^{N}} \frac{1}{2}\left[\left|\nabla_{\xi} v(\xi)\right|^{2}+W(v(\xi))\right]=h^{N \beta-\alpha} J(v) .
\end{aligned}
$$

We can now describe the concentration properties of the modulus of solution of (29).

Lemma 4.3 For any $\varepsilon>0$, there exist positive numbers $\Delta=\Delta(\varepsilon), \widehat{R}=\widehat{R}(\varepsilon)$ such that for any $\psi(t, x)$ that solves (29), with $\left|\psi\left(t, h^{\beta} x\right)\right| \in J^{m+\Delta} \cap S_{\sigma}$, for all $t$, there exists a map $\widehat{q}_{h}: \mathbb{R}_{0}^{+} \rightarrow \mathbb{R}^{N}$ for which

$$
\frac{\int_{\mathbb{R}^{N} \backslash B\left(\widehat{q}_{h}(t), \widehat{R}\right)}\left|\nabla u_{h}(t, x)\right|^{2} d x}{\int_{\mathbb{R}^{N}}\left|\nabla u_{h}(t, x)\right|^{2} d x}<\varepsilon .
$$

Proof For fixed $h$ and $t$, we set $v(\xi)=\left|\psi\left(t, h^{\beta} \xi\right)\right|$. By Lemma 4.2, there exist $\Delta>0$, $\widehat{R}>0$ and $\bar{q}=\bar{q}(v)$ such that if $\left|\psi\left(t, h^{\beta} x\right)\right| \in J^{m+\Delta} \cap S_{\sigma}$, then

$$
\varepsilon>\frac{\int_{\mathbb{R}^{N} \backslash B(\bar{q}, \widehat{R})}\left|\nabla_{\xi} v(\xi)\right|^{2} d \xi}{\int_{\mathbb{R}^{N}}\left|\nabla_{\xi} v(\xi)\right|^{2} d \xi} .
$$

By a change of variables, we obtain

$$
\begin{aligned}
\varepsilon & >\frac{\int_{\mathbb{R}^{N} \backslash B(\bar{q}, \widehat{R})}\left|\nabla_{\xi} v(\xi)\right|^{2} d \xi}{\int_{\mathbb{R}^{N}}\left|\nabla_{\xi} v(\xi)\right|^{2} d \xi}=\frac{\int_{\mathbb{R}^{N} \backslash B\left(h^{\beta} \bar{q}, h^{\beta} \widehat{R}\right)}\left|\nabla u_{h}(t, x)\right|^{2} d x}{\int_{\mathbb{R}^{N}}\left|\nabla u_{h}(t, x)\right|^{2} d x} \\
& >\frac{\int_{\mathbb{R}^{N} \backslash B\left(h^{\beta} \bar{q}, \widehat{R}\right)}\left|\nabla u_{h}(t, x)\right|^{2} d x}{\int_{\mathbb{R}^{N}}\left|\nabla u_{h}(t, x)\right|^{2} d x} \quad \text { since } h<1 .
\end{aligned}
$$

Setting $\widehat{q}_{h}(t)=h^{\beta} \bar{q}$, we complete the proof. Notice that $\widehat{q}_{h}(t)$ depends on $\varepsilon$, and $\psi$, while $\widehat{R}$ depends only on $\varepsilon$. 
Proposition 4.4 Let $V \in L_{\mathrm{loc}}^{\infty}$. For every $\varepsilon>0$, there exist $\widehat{R}>0$ and $h_{0}>0$ such that, for any $\psi(t, x)$ that solves (29), with initial data $\psi(0, x) \in B_{K, h}$, where $h<h_{0}$, and for any $t$, there exists $\widehat{q}_{h}(t) \in \mathbb{R}^{N}$, for which

$$
\frac{1}{\left\|\nabla u_{h}(t, x)\right\|_{L^{2}}^{2}} \int_{\mathbb{R}^{N} \backslash B\left(\widehat{q}_{h}(t), h^{\beta} \widehat{R}\right)}\left|\nabla u_{h}(t, x)\right|^{2} d x<\varepsilon .
$$

Proof By the conservation law, the energy $E_{h}(\psi(t, x))$ is constant with respect to $t$. Then we have

$$
\begin{aligned}
E_{h}(\psi(t, x)) & =E_{h}(\psi(0, x)) \\
& =\widetilde{J}_{h}\left(u_{h}(0, x)\right)+\int_{\mathbb{R}^{N}} u_{h}^{2}(0, x)\left[\frac{\left|\nabla s_{h}(0, x)\right|^{2}}{2}+V(x)\right] d x \\
& \leqslant \widetilde{J}_{h}\left(u_{h}(0, x)\right)+\frac{K}{2} \sigma^{2} h^{N \beta}+K h^{N \beta} \\
& \leqslant h^{N \beta-\alpha} J(U+w)+h^{N \beta} C,
\end{aligned}
$$

where $C$ is a suitable constant. By rescaling, and using that $\psi(0, x) \in B_{K, h}$, and that $\|w\|_{H^{1}}<K h^{\alpha}$ implies $J(U+w)<m+K h^{\alpha}$ (see the proof of Proposition 3.10), we obtain

$$
\begin{aligned}
E_{h}(\psi(t, x)) & =h^{N \beta-\alpha} J(U+w)+h^{N \beta} C \\
& <h^{N \beta-\alpha}\left(m+K h^{\alpha}\right)+h^{N \beta} C \\
& =h^{N \beta-\alpha}\left(m+K h^{\alpha}+h^{\alpha} C\right)=h^{N \beta-\alpha}\left(m+h^{\alpha} C_{1}\right),
\end{aligned}
$$

where we have set $C_{1}=K+C$. Thus

$$
\begin{aligned}
\widetilde{J}_{h}\left(u_{h}(t, x)\right) & =E_{h}(\psi(t, x))-\int_{\mathbb{R}^{N}} u_{h}^{2}(t, x)\left[\frac{\left|\nabla s_{h}(t, x)\right|^{2}}{2}+V(x)\right] d x \\
& <h^{N \beta-\alpha}\left(m+h^{\alpha} C_{1}\right),
\end{aligned}
$$

since $V \geqslant 0$. By rescaling inequality (35), we get

$$
J\left(u_{h}\left(t, h^{\beta} x\right)\right)<m+h^{\alpha} C_{1} .
$$

So, for $h$ sufficiently small, we may apply Lemma 4.3, and get the claim. 


\subsection{Case $\Omega$ is bounded}

The case where $\Omega$ is bounded is easily treated, once one makes the crucial remark that Lemma 4.2 has to replace [6, Lemma 15], that it was used in the proof of Lemma 3.8. The rest of the proofs in the consequent Lemmas 4.5 and 4.6 and of the final Proposition 4.7 follow precisely the pattern of the proofs for Lemmas 3.8, 3.9, and of Proposition 3.10, respectively. For completeness, we give below the precise statements, where we have assumed for simplicity, as in the $L^{2}$ case, that $\sigma=1$.

Lemma 4.5 Forany $\varepsilon>0$, there exist $\Delta=\Delta(\varepsilon), h_{0}=h_{0}(\varepsilon)>0$ and $R=R(\varepsilon)>0$ such that, for any $0<h<h_{0}(\varepsilon)$, there is an open ball $B\left(\widehat{q}_{h}, h^{\beta} R\right) \subset \Omega$ so that for any $u \in H_{0}^{1}(\Omega)$ with $C_{h}(u)=1$, and $J_{h}(u)<m(h, \Omega)+\Delta h^{-\alpha}$,

$$
\frac{\int_{\Omega \backslash B\left(\widehat{q}_{h}, h^{\beta} R\right)}|\nabla u|^{2} d x}{\int_{\Omega}|\nabla u|^{2} d x}<\varepsilon .
$$

Lemma 4.6 Forany $\varepsilon>0$, there exist $\Delta=\Delta(\varepsilon), h_{0}=h_{0}(\varepsilon)>0$ and $R=R(\varepsilon)>0$ such that, for any $0<h<h_{0}(\varepsilon)$, there is an open ball $B\left(\widehat{q}_{h}, h^{\beta} R\right) \subset \Omega$ so that for a solution $\psi(t, x)$ of $(1)$ with $C_{h}(|\psi(t, x)|)=1$, and $J_{h}(|\psi(t, x)|)<m(h, \Omega)+\Delta h^{-\alpha}$, for each $t \in \mathbb{R}_{0}^{+}$,

$$
\frac{\int_{\Omega \backslash B\left(\widehat{q}_{h}, h^{\beta} R\right)}|\nabla u(t, x)|^{2} d x}{\int_{\Omega}|\nabla u(t, x)|^{2} d x}<\varepsilon,
$$

where $u(t, x)=|\psi(t, x)|$.

Proposition 4.7 Given $\varepsilon>0$, there exist $h_{0}=h_{0}(\varepsilon)>0$ and $R=R(\varepsilon)>0$ such that, for any $0<h<h_{0}(\varepsilon)$, there is an open ball $B\left(\widehat{q}_{h}, h^{\beta} R\right) \subset \Omega$ so that for a solution $\psi(t, x)$ of $(1)$ with $C_{h}(|\psi(t, x)|)=1$, and with initial data $\psi(0, x) \in B_{h}^{K}$, where $K$ is a positive fixed number,

$$
\frac{\int_{\Omega \backslash B\left(\widehat{q}_{h}, h^{\beta} R\right)}|\nabla u(t, x)|^{2} d x}{\int_{\Omega}|\nabla u(t, x)|^{2} d x}<\varepsilon,
$$

for any $t \in \mathbb{R}_{0}^{+}$, where $u(t, x)=|\psi(t, x)|$.

\section{Appendix}

In order to gain some control over dynamics of the problem, that is, to try to formulate Newton's equation describing the motion of the barycenter for a fixed value of $h$, one needs to express suitably $\ddot{q}(t)$. To this end, a further assumption on $W$ is made, namely, that $W(0)=0$. For the sake of simplicity, we fix $h=1, \alpha=1$ in what follows, the general case is straightforward. Also, we suppose that a solution $\psi(t, x)$ is sufficiently smooth in order to make the integration by parts meaningful. Given 
this, the general case can be proved with minor technical efforts. Finally, we use the Einstein convention on the summation indices.

We will use the Lagrangian formalism. Equation (1) is the Euler-Lagrange equation relative to the following Lagrangian density $\mathcal{L}$ :

$$
\mathcal{L}=\operatorname{Re}\left(i \bar{\psi} \partial_{t} \psi\right)-\frac{1}{2}|\nabla \psi|^{2}-W(|\psi|)-V(x)|\psi|^{2}
$$

By Noether's theorem, there are continuity equations related to $\mathcal{L}$, which we will use to derive an equation for the motion. In particular, we are interested in the following continuity equations:

$$
\begin{aligned}
\frac{d}{d t}|\psi(t, x)|^{2} & =-\nabla \cdot \operatorname{Im}(\bar{\psi} \nabla \psi), \\
\frac{d}{d t} \operatorname{Im}(\bar{\psi} \nabla \psi) & =-|\psi|^{2} \nabla V-\nabla \cdot T,
\end{aligned}
$$

where $T$ is the so-called energy stress tensor and has the form

$$
T_{j k}=\operatorname{Re}\left(\partial_{x_{j}} \psi \partial_{x_{k}} \bar{\psi}\right)-\Delta_{j k}\left[\operatorname{Re}\left(\frac{1}{2} \bar{\psi} \Delta \psi\right)+\frac{1}{2}|\nabla \psi|^{2}-\frac{1}{2} W^{\prime}(|\psi|)|\psi|+W(|\psi|)\right]
$$

For an introduction to the Lagrangian formalism for equation (1) and continuity equations we refer to $[5,8,16]$.

In the light of equation (36), and by divergence theorem, one has for $j=1, \ldots, N$,

$$
\begin{aligned}
\dot{q}_{j}(t) & =\frac{d}{d t} \int_{\Omega} x_{j}|\psi(t, x)|^{2} d x=\int_{\Omega} x_{j} \partial_{t}\left(|\psi|^{2}\right) d x \\
& =-\int_{\Omega} x_{j} \nabla \cdot \operatorname{Im}(\bar{\psi} \nabla \psi) d x \\
& =-\int_{\Omega} \nabla \cdot\left[x_{j} \operatorname{Im}(\bar{\psi} \nabla \psi)\right]+\int_{\Omega} \operatorname{Im}\left(\bar{\psi} \partial_{x_{j}} \psi\right) \\
& =\int_{\Omega} \operatorname{Im}\left(\bar{\psi} \partial_{x_{j}} \psi\right),
\end{aligned}
$$

since $\psi(t, x)=0$ on $\partial \Omega$. Thus we have the momentum law

$$
\dot{q}(t)=\operatorname{Im} \int_{\Omega} \bar{\psi} \nabla \psi d x
$$

For the second derivative of the center of mass, we have, by (37) and by divergence theorem, 


$$
\begin{aligned}
\ddot{q}_{j}(t) & =\frac{d}{d t} \int_{\Omega} \operatorname{Im} \bar{\psi} \partial_{x_{j}} \psi d x \\
& =-\int_{\Omega} \partial_{x_{k}} T_{j k}(t, x) d x-\int_{\Omega}|\psi(t, x)|^{2} \partial_{x_{j}} V(x) d x \\
& =\int_{\partial \Omega} T_{j k}(t, x) \cdot n_{k} d \sigma-\int_{\Omega}|\psi(t, x)|^{2} \partial_{x_{j}} V(x) d x=\mathrm{I}_{1}+\mathrm{I}_{2},
\end{aligned}
$$

where $n$ is the inward normal to $\partial \Omega$.

Let us use the polar form $\psi(t, x)=u(t, x) e^{i s(t, x)}$. Then

$$
\mathrm{I}_{2}=-\int_{\Omega} u^{2} \partial_{x_{j}} V(x) d x
$$

This appears to be a force term depending on the potential $V$. This, when the concentration parameter $h \rightarrow 0$, gives us the Newtonian law for the motion of a particle (see [6], where this approach is used in the whole space $\mathbb{R}^{N}$ ).

Since $u=0$ on the boundary (and since $W(0)=0$ ), the expression of $T$ is simplified and the term $\mathrm{I}_{1}$ becomes

$$
\begin{aligned}
\mathrm{I}_{1} & =\int_{\partial \Omega} T_{j k} n_{k} d \sigma=\int_{\partial \Omega}\left(\partial_{x_{j}} u \partial_{x_{k}} u-\frac{1}{4} \Delta_{j k} \Delta\left(u^{2}\right)\right) n_{k} d \sigma \\
& =\int_{\partial \Omega}\left(\partial_{x_{j}} u \partial_{x_{k}} u-\frac{1}{2} \Delta_{j k}|\nabla u|^{2}\right) n_{k} d \sigma .
\end{aligned}
$$

Again, because $u=0$ on the boundary, by the implicit function theorem, we have that $\nabla u$ is orthogonal to $\partial \Omega$. In addition, we have by defintion $u=|\psi| \geqslant 0$, so whenever $\nabla u \neq 0$ the inward pointing normal vector can be written as $n=\nabla u /|\nabla u|$. Thus

$$
\begin{aligned}
\mathrm{I}_{1} & =\int_{\partial \Omega}\left(\partial_{x_{j}} u \partial_{x_{k}} u-\frac{1}{2} \Delta_{j k}|\nabla u|^{2}\right) \frac{\partial_{x_{k}} u}{|\nabla u|} d \sigma \\
& =\int_{\partial \Omega}\left(\partial_{x_{j}} u|\nabla u|-\frac{1}{2} \partial_{x_{j}} u|\nabla u|\right) d \sigma \\
& =\frac{1}{2} \int_{\partial \Omega} \partial_{x_{j}} u|\nabla u| d \sigma=\frac{1}{2} \int_{\partial \Omega}|\nabla u|^{2} n_{j} d \sigma .
\end{aligned}
$$

Concluding, we have

$$
\ddot{q}(t)=-\int_{\Omega} u^{2} \nabla V(x) d x+\frac{1}{2} \int_{\partial \Omega}|\nabla u|^{2} n d \sigma .
$$

In the case of a bounded domain with Dirichlet boundary condition, there appears an extra term, which represents the centripetal force. Unfortunately, there are some obvious computational challenges concerning the last integral of (38), and we cannot give a simple expression of this term when $h \rightarrow 0$. As it was said in Introduction, these challenges call for further work on dynamics of solution of (1). 
Acknowledgments Manolis Magiropoulos wishes to thank Georgios M. Kavoulakis and John Smyrnakis for many helpful discussions. The authors want also to thank the referee for his/her useful remarks and comments.

\section{References}

1. Ambrosetti, A., Badiale, M., Cingolani, S.: Semiclassical states of nonlinear Schrödinger equations. Arch. Ration. Mech. Anal. 140(3), 285-300 (1997)

2. Ambrosetti, A., Malchiodi, A., Secchi, S.: Multiplicity results for some nonlinear Schrödinger equations with potentials. Arch. Ration. Mech. Anal. 159(3), 253-271 (2001)

3. Bellazzini, J., Benci, V., Bonanno, C., Sinibaldi, E.: Hylomorphic solitons in the nonlinear KleinGordon equation. Dyn. Partial Differ. Equ. 6(4), 311-334 (2009)

4. Bellazzini, J., Benci, V., Ghimenti, M., Micheletti, A.M.: On the existence of the fundamental eigenvalue of an elliptic problem in $\mathbb{R}^{N}$. Adv. Nonlinear Stud. 7(3), 439-458 (2007)

5. Benci, V.: Hylomorphic solitons. Milan J. Math. 77, 271-332 (2009)

6. Benci, V., Ghimenti, M., Micheletti, A.M.: The nonlinear Schroedinger equation: solitons dynamics. J. Differential Equations 249, 3312-3341 (2010)

7. Benci, V., Ghimenti, M., Micheletti, A.M.: The nonlinear Schroedinger equation: existence, stability and dynamics of solutions. In: Liu, C., Long, Y. (eds.) Progress in Variational Methods. Nankai Series in Pure, Applied Mathematics and Theoretical Physics, vol. 7, pp. 11-29. World Scientific, Hackensack (2011)

8. Benci, V., Ghimenti, M., Micheletti, A.M.: On the dynamics of solitons in the nonlinear Schrödinger equation. Arch. Ration. Mech. Anal. 205(2), 467-492 (2012)

9. Benci, V., Grisanti, C.R., Micheletti, A.M.: Existence and non existence of the ground state solution for the nonlinear Schroedinger equations with $V(\infty)=0$. Topol. Methods Nonlinear Anal. 26(2), 203-219 (2005)

10. Cao, D., Dancer, N.E., Noussair, E.S., Yan, S.: On the existence and profile of multi-peaked solutions to singularly perturbed semilinear Dirichlet problems. Discrete Contin. Dynam. Systems 2(2), 221-236 (1996)

11. Cazenave, T.: Semilinear Schrödinger Equations. Courant Lecture Notes in Mathematics, vol. 10. Courant Institute of Mathematical Sciences, New York (2003)

12. Cazenave, T., Lions, P.-L.: Orbital stability of standing waves for some nonlinear Schrödinger equations. Comm. Math. Phys. 85(4), 549-561 (1982)

13. Cingolani, S., Lazzo, M.: Multiple semiclassical standing waves for a class of nonlinear Schrödinger equations. Topol. Methods Nonlinear Anal. 10(1), 1-13 (1997)

14. del Pino, M., Felmer, P.L.: Local mountain passes for semilinear elliptic problems in unbounded domains. Calc. Var. Partial Differential Equations 4(2), 121-137 (1996)

15. Floer, A., Weinstein, A.: Nonspreading wave packets for the cubic Schrödinger equation with a bounded potential. J. Funct. Anal. 69(3), 397-408 (1986)

16. Gelfand, I.M., Fomin, S.V.: Calculus of Variations. Prentice-Hall, Englewood Cliffs (1963)

17. Ginibre, J., Velo, G.: On a class of nonlinear Schrödinger equations. II. Scattering theory, general case. J. Funct. Anal. 32(1), 33-71 (1979)

18. Kato, T.: Nonlinear Schrödinger equations. In: Holden, H., Jensen, A. (eds.) Schrödinger Operators. Lecture Notes in Physics, vol. 345, pp. 218-263. Springer, Berlin (1989)

19. Li, Y.Y.: On a singularly perturbed elliptic equation. Adv. Differential Equations 2(6), 955-980 (1997)

20. Ni, W.-M., Wei, J.: On the location and profile of spike-layer solutions to singularly perturbed semilinear Dirichlet problems. Comm. Pure Appl. Math. 48(7), 731-768 (1995)

21. Noris, B., Tavares, H., Verzini, G.: Existence and orbital stability of the ground states with prescribed mass for the $L^{2}$-critical and supercritical NLS on bounded domains. Anal. PDE 7(8), 1807-1838 (2014)

22. Oh, Y.-G.: Existence of semiclassical bound states of nonlinear Schrödinger equations with potentials of the class $(V)_{a}$. Comm. Partial Differential Equations 13(12), 1499-1519 (1988)

23. Rabinowitz, P.H.: On a class of nonlinear Schrödinger equations. Z. Angew. Math. Phys. 43(2), 270291 (1992)

24. Wei, J.: On the construction of single-peaked solutions to a singularly perturbed semilinear Dirichlet problem. J. Differential Equations 129(2), 315-333 (1996) 